\title{
The Use of Omalizumab in Food Oral Immunotherapy
}

\author{
Roxane Labrosse $^{1} \cdot$ François Graham $^{2} \cdot$ Anne Des Roches $^{1} \cdot$ Philippe Bégin $^{1,2}$ (I)
}

Received: 2 February 2016/ Accepted: 4 July 2016/Published online: 14 September 2016

(c) L. Hirszfeld Institute of Immunology and Experimental Therapy, Wroclaw, Poland 2016

\begin{abstract}
Food allergy is an important health issue that affects up to $8 \%$ of the population. The management of allergic patients involves allergen avoidance and prompts the treatment of accidental reactions, as no curative treatment is available so far in routine practice. Oral immunotherapy (OIT) is a promising therapeutic alternative, but it is associated with frequent allergic reactions and cost-effectiveness issues. In hopes of reducing such reactions, a number of trials have used omalizumab, an anti-IgE monoclonal humanized antibody, as adjunctive therapy in OIT. The allergens studied in these omalizumab-enabled OIT trials include peanuts, milk, eggs, or mixes of multiple foods. In this article, we review the major findings from these studies and discuss potential benefits and issues related to omalizumab-enabled OIT. Results from the previous trials suggest that the use of omalizumab could potentially lead to safer and more efficient OIT protocols, by reducing the number and severity of reactions, and increasing allergen tolerance threshold. While more evidence is needed with regard to the maintenance of the longterm tolerance after OIT, omalizumab's potential immunomodulatory role could be of benefit. More studies are needed to further document this new indication for omalizumab.
\end{abstract}

Philippe Bégin

philippe.begin@umontreal.ca

1 Pediatric Allergy and Clinical Immunology, CHU SainteJustine, University of Montreal, Montreal, QC, Canada

2 Allergy and Clinical Immunology, CHUM, University of Montreal, Montreal, QC, Canada
Keywords Immunotherapy - Desensitization · Oral immunotherapy · Food allergy · Omalizumab · Anti-IgE therapy

\section{Introduction}

Food allergy is a common condition that affects up to $8 \%$ of the population (Gupta et al. 2011; McGowan and Keet 2013; Soller et al. 2012), with a highest prevalence amongst young children, and is expected to increase with time (Sicherer and Sampson 2010). It also represents a significant economic burden for both society and families (Gupta et al. 2013). The main recognized allergens in North America are peanuts, tree nuts, cow's milk, soy, fish, and shellfish (Boyce et al. 2010). No treatment is currently available in routine practice: strict avoidance of the offending allergen and carrying auto-injectable epinephrine in the case of inadvertent exposure are the standard of care (Lanser et al. 2015; Sicherer and Sampson 2010). However, this can significantly affect the quality of life of patients and families living in fear of accidental exposure (Cummings et al. 2010; Sicherer et al. 2001), and leave children at risk of nutritional deficiencies (Sova et al. 2013).

\section{Oral Immunotherapy to Treat Food Allergy}

Research is underway to identify potential therapeutic avenues for food allergy. In the past decade, food immunotherapy has been developed in hope of eliminating food-related reactions in allergic patients (Sato et al. 2014). Different approaches to food immunotherapy have been attempted: subcutaneous, epicutaneous, sublingual, and finally oral immunotherapy (OIT). The latter is the best- 
studied approach and has been shown to induce desensitization effectively. Despite variations between protocols, OIT usually takes place in three phases: initial dose escalation, build-up phase, and maintenance phase (Chhiba et al. 2015).

Oral immunotherapy to food has been successful in numerous trials, namely, in desensitization to peanuts (Beyer and Wahn 2008; Blumchen et al. 2010; Clark et al. 2009; Hofmann et al. 2009; Jones et al. 2009; Varshney et al. 2011), eggs (Patriarca et al. 2003; Buchanan et al. 2007; Staden et al. 2007; Burks et al. 2012), milk (Longo et al. 2008; Meglio et al. 2004; Patriarca et al. 2003, Skripak et al. 2008, Staden et al. 2007, 2008), and to multiple foods (including peanuts, tree nuts, sesame, dairy, and eggs) (Begin et al. 2014b). However, there is still progress to be made, as $10-30 \%$ of patients are refractory to OIT, especially those with very high food-specific IgE (Schneider et al. 2013; Umetsu et al. 2015). In addition, many require prolonged desensitization therapy before reaching maintenance dose, ranging from many months to years [median time ranging 20-60 weeks (Umetsu et al. 2015), and up to 85 weeks in multi-food protocols (Begin et al. 2014b)]. Finally, the achieved dose is often below the targeted dose (Umetsu et al. 2015), potentially protecting patients in the case of accidental exposure, but not allowing them to consume normal amounts of the culprit food in their diet.

\section{Omalizumab as an Adjunct to Oral Immunotherapy}

Efforts to improve safety and, therefore, efficacy of OIT have included the use of anti-histamines, such as cetirizine (Lafuente et al. 2014; Meglio et al. 2004), mast cell stabilizers, such as ketotifen (Jagdis et al. 2014), or the leukotriene receptor antagonist montelukast (Takahashi et al. 2014), with the modest success. More recently, the anti-IgE molecule omalizumab has been used to accelerate OIT desensitization in high-risk patients and to improve the safety profile of this procedure.

Omalizumab is a monoclonal humanized antibody that targets $\operatorname{IgE}$ and has been licensed for use in asthma refractory to inhaled corticosteroids and chronic idiopathic urticaria. To prime a type I hypersensitivity reaction, IgE molecules must first bind to Fc receptors (FceRI) found on the surface of mast cells and basophils. The main purpose of anti-IgE therapy is to reduce concentrations of circulating $\operatorname{IgE}$ and ultimately sequester $\operatorname{IgE}$ from FceRI on target cells (Chang and Shiung 2006; Segal et al. 2008), thus preventing mast cell activation. An important characteristic of omalizumab is that it does not bind to $\operatorname{IgE}$ already bound to FceRI on mastocytes and basophils (Wright et al. 2015), which is crucial as such binding could induce cross-linking and activation of FceRI, and potentially lead to anaphylactic reactions upon injection.

This review will focus on the summary and analysis of OIT trials using omalizumab as adjuvant therapy and then discuss cutting-edge issues regarding this approach.

\section{Omalizumab Trials in Food Immunotherapy}

The first study exploring the potential of anti-IgE therapy in food allergy was conducted with talizumab (TNX-901), an analog of omalizumab no longer available on the market. The study was novel in that it showed an increased tolerance threshold during oral challenges in peanut-allergic patients (Leung et al. 2003). While such results were promising, a phase II trial using omalizumab was ended prematurely due to two anaphylactic reactions that occurred early on during food challenges, limiting the power of the study (Sampson et al. 2011). Nonetheless, the study showed similar trends. Thereafter, interest was shifted towards exploiting these benefits in other clinical contexts, namely, in OIT, and many studies have since then been conducted evaluating the use of anti-IgE molecules in this setting (Table 1).

\section{OIT Trials Using Omalizumab as Adjuvant Therapy}

\section{Peanuts}

Peanut allergy affects more than $1 \%$ of the population (Ben-Shoshan et al. 2009; Sicherer et al. 2003) and tends to persist over time (Skolnick et al. 2001). Accidental exposure to peanuts are frequent (Yu et al. 2006), and they are the most common trigger in fatal anaphylactic reactions to food (Bock et al. 2001). Peanut OIT trials have, therefore, generated much interest, although they have shown limitations as previously discussed.

A recent study by Schneider et al. (2013) evaluated the use of omalizumab as adjunctive therapy in an oral peanut desensitization protocol in high-risk peanut-allergic subjects. Thirteen pediatric patients (median age 10 years, range $8-16$ years) were enrolled in the study. Patients received a 12-week course of omalizumab (dosage according to European dosing guidelines) before being admitted for initial rush oral desensitization, which consisted in a gradual increase up to $500 \mathrm{mg}$ of peanut flour (cumulative dose of $992 \mathrm{mg}$ ) over $6 \mathrm{~h}$. Patients then continued with a slower weekly escalation over the next 8 weeks (weeks 12-20) up to a final maintenance dose of $4000 \mathrm{mg}$ of peanut flour, after which omalizumab was discontinued while daily oral peanut ingestion was maintained. A second DBPCFC was conducted 12 weeks after 


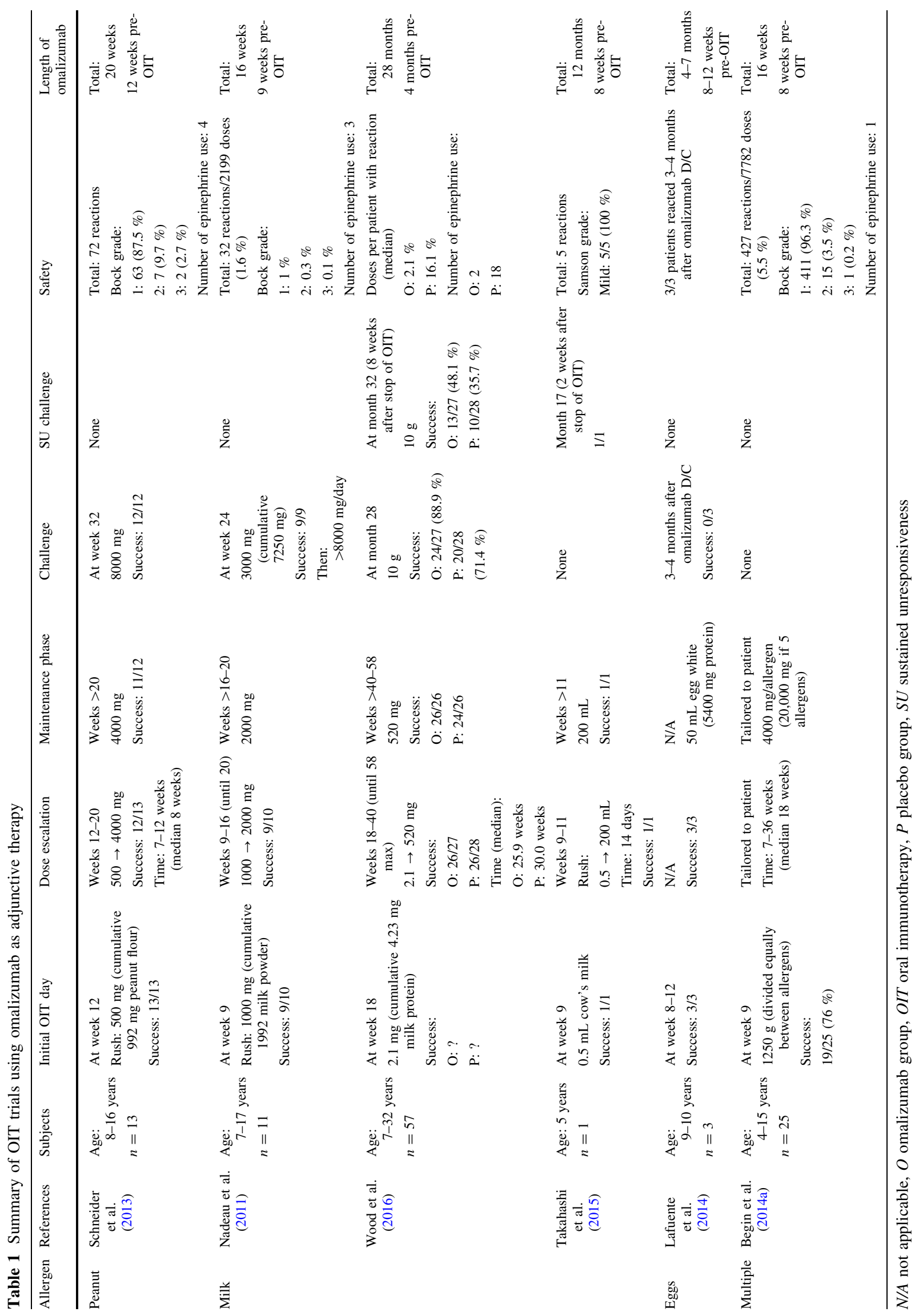


omalizumab was stopped. Patients were followed for a total of 52 weeks.

Interestingly, all 13 patients (100\%) were able to reach the initial $500 \mathrm{mg}$ of peanut flour (cumulative dose of $992 \mathrm{mg}$ ) on the first day with minimal or no symptoms, as shown by $7 / 13$ (54\%) of the patients having no reactions, and the remaining six developing mild (Grade 1) reactions, only two of which were treated with anti-histamines.

During the following escalation weeks, 12 of the 13 children $(92 \%)$ were then able to tolerate the $4000 \mathrm{mg}$ dose within a median time of 8 weeks (range 7-12 weeks). During this up-dosing phase, 49 reactions occurred (96\% Grade 1 and $4 \%$ Grade 2), mostly nausea, hypersalivation, and abdominal pain.

Of the 12 participants that remained in the study, all 12 (100\%) eventually passed an oral food challenge (OFC) with a cumulative dose of $8000 \mathrm{mg}$ of peanut flour, the equivalent of approximately 20 peanuts (and 160-400 times the dose tolerated before desensitization). Following omalizumab discontinuation and while on maintenance dosing, there were 17 reactions recorded during the 6 months observation period, two of which were severe (Grade 3), with four epinephrine uses at home in two subjects.

A total of 72 reactions were recorded during the entire period of the study, a third of which occurred in the same subject. The reactions were mostly mild, and mainly of gastro-intestinal origin (nausea and hypersalivation). Some of these symptoms faded after peanut flour was switched to capsules, indicating a possible peanut aversion instead of true allergic reaction. Nearly half of the patients $(6 / 13$, $46 \%$ ) experienced mild or no reactions, and almost a quarter $(3 / 13,23 \%)$ had no reaction at all during the time of the study. No severe reactions (Grade 3) occurred while on omalizumab, suggesting that omalizumab might have a protective effect on such events.

\section{Milk}

About 2-3\% of young children are allergic to cow's milk, making it one of the most common food allergies in this age group (Boyce et al. 2010; Sicherer 2011). While the majority of children will outgrow their milk allergy, spontaneous recovery in older children with very high $\operatorname{IgE}$ levels is less likely (Garcia-Ara et al. 2004; Shek et al. 2004). Moreover, cow's milk is a staple food that is widely present in Western diets, and its avoidance has a negative impact on the quality of life (Protudjer et al. 2015).

Nadeau et al. studied 11 milk-allergic patients treated with omalizumab during rapid OIT (Nadeau et al. 2011). After 9 weeks of omalizumab therapy, rapid OIT was performed successfully in nine out of ten subjects with a gradual increase of milk powder up to a maximum dose of
$1000 \mathrm{mg}$ (cumulative dose, $1992 \mathrm{mg}$ ). One of these subjects, however, received epinephrine after completing his challenge for refractory nasal obstruction and urticaria. The ten subjects continued with daily home dosing and weekly increases of milk powder doses over the following 7-11 weeks until they reached $2000 \mathrm{mg}$, which was achieved successfully in nine out of the ten patients. Omalizumab was discontinued at week 16, and a DBPCFC of $3000 \mathrm{mg}$ (cumulative dose of $7250 \mathrm{mg}$ ) was performed at week 24 , followed by an open challenge of $4000 \mathrm{mg}$ the same day $(n=4)$ or of $8000 \mathrm{mg}$ the next day $(n=5)$. All nine patients who tolerated $2000 \mathrm{mg}$ daily dosing passed the OFC and continued with a minimal daily milk ingestion of $8000 \mathrm{mg}$ (equivalent of $\geq 240 \mathrm{~mL}$ of milk). Out of the 2199 doses administered, 32 resulted in subsequent reactions (1.6\% of total doses), and only $0.1 \%$ of these were considered severe.

A more recent study by Wood et al. (2016) evaluated the benefits of using a more prolonged course of omalizumab, while OIT was performed for milk-allergic patients, using a randomized, double-blind, placebo-controlled approach. In this study, 57 patients (age 7-32 years) were randomized to either placebo or omalizumab therapy (randomization 1:1). They received a blinded treatment of either omalizumab or placebo injections every 2 or 4 weeks during the first 18 weeks, after which time OIT was started. Subsequently, the doses were escalated every 2 weeks during 22-40 weeks, reaching a minimum maintenance dose of $520 \mathrm{mg}$ of milk protein, the equivalent to $15 \mathrm{~mL}$ of liquid milk. At month 16, the treatment was unblinded, and patients in the placebo arm stopped injections while patients under omalizumab therapy continued for an additional 12 months, followed by omalizumab discontinuation. At month 28, patients from both arms underwent an OFC of $10 \mathrm{~g}$. Those having passed this challenge then continued immunotherapy for another 8 weeks, followed by avoidance of allergen for 8 weeks, and then underwent a $10 \mathrm{~g}$ sustained unresponsiveness OFC at month 32.

There were no significant differences between the two groups in terms of successful $10 \mathrm{~g}$ OFC at week 28 ( $88.9 \%$ in the omalizumab group vs. $71.4 \%$ in the placebo group; $p=0.18$ ), nor in the $10 \mathrm{~g}$ sustained unresponsiveness OFC at month $32(48.1 \%$ in the omalizumab group vs. $35.7 \%$ in the placebo group; $p=0.42$ ). However, omalizumab-treated patients had fewer adverse symptoms during the escalation period (median percentage of doses per subject with symptoms of 2.1 vs. $16.1 \%$ ) and required less treatment (median dose-related reactions requiring treatment of 0.0 vs. $3.8 \%$ ). Although not considered statistically significant $(p=0.052)$, there was also a trend towards less use of epinephrine in the omalizumab group: out of the 40,641 doses administered, 20 required 
epinephrine, only two of which were in the omalizumabtreated patients. This study failed to show a statistically significant improvement in desensitization success and sustained unresponsiveness in patients treated with omalizumab during OIT, although it may have been underpowered to do so. However, it demonstrated noticeable benefits in terms of safety (less reactions) and efficiency (maintenance dose achieved faster).

A recent case report also illustrates the potential benefits of using omalizumab for a longer period of time (Takahashi et al. 2015). This report describes a 5 year-old boy with recurrent anaphylaxis to milk and OFC-proven milk allergy that was treated with a rush OIT protocol $(200 \mathrm{~mL}$ of cow's milk over 14 days) after 8 weeks of omalizumab treatment. During the rush escalation phase, only one reaction occurred, and five reactions occurred in total during the length of the study, all of which were mild. Omalizumab was stopped after a year, at which point skin prick tests had become negative, and home milk dosing was continued for another 5 months. A sustained unresponsiveness challenge was performed afterwards, after 2 weeks of milk avoidance, and was negative.

However, prolonged treatment might be costly and more studies are needed to prove its benefits over a shorter treatment, as there are some reports of patients successfully desensitized to milk with an omalizumab-assisted OIT without any recurrence of allergic symptoms even after omalizumab discontinuation (Lafuente et al. 2014), in addition to the studies previously discussed (Nadeau et al. 2011; Wood et al. 2016).

\section{Eggs}

Prevalence of egg allergy is about 1-2 \% in the pediatric population, and it is one of the major childhood food allergens (Eggesbo et al. 2001; Gupta et al. 2011; Soller et al. 2012).

As of today, there are no clinical trials that have studied omalizumab-enabled OIT in egg allergic patients. However, Lafuente et al. (2014) report a case series of three patients who successfully underwent egg OIT with omalizumab as adjunctive therapy, but developed a recurrence of symptoms once omalizumab therapy was discontinued. Two of the three patients had failed initial non-omalizumab-assisted OIT, and it was anticipated that the third would also be unable to complete the initial OIT. After 2-3 months of omalizumab therapy, all were able to gradually tolerate $50 \mathrm{~mL}$ of raw egg white $(5400 \mathrm{mg}$ of protein) and two passed an omelet challenge uneventfully. The patients were then instructed to eat eggs minimally three times per week. Omalizumab was continued for another 2-4 months after maintenance dose was reached. Three $(n=2)$ to four $(n=1)$ months after omalizumab was stopped, these three patients experienced predominantly gastro-intestinal symptoms (vomiting, abdominal pain) after eating egg and failed a subsequent egg challenge. They were put on omalizumab for another two months, after which two of the patients successfully passed an open challenge (the third one being scheduled for the challenge at the time of the study). There is mention of two more patients who similarly could not tolerate OIT without the help of omalizumab therapy.

Omalizumab could, therefore, be particularly helpful in high-risk patients, and these patients might benefit from a longer course of omalizumab therapy. However, due to the small number of patients and the fact that the daily amount of allergen ingested could vary greatly (none vs. ad lib, with a minimal consumption of three times/week), these results need to be interpreted with caution. One can speculate that if patients had been put on a regular daily maintenance dose as in other published trials of omalizumab-enabled OIT, these subjects might not have been re-sensitized.

\section{Multiple Foods}

It is estimated that $30 \%$ of food-allergic children have allergies to multiple foods (Gupta et al. 2011), which can significantly affect their quality of life (Sicherer et al. 2001). In the interest of diminishing both psychological and economic burdens for these families, a multiple foods OIT protocol has successfully been established with a safety profile comparable to single-allergen desensitization (Begin et al. 2014b). Although faster than desensitizing each allergen sequentially, the protocol remained laborious with a median time of 85 weeks (range 54-156) to reach the maintenance dose of $4000 \mathrm{mg}$ protein per allergen.

Begin et al. (2014a) conducted a phase 1 study evaluating the tolerability and safety of a rush OIT to multiple foods using omalizumab as adjunctive therapy. Twentyfive patients were enrolled in the study, with ages ranging 4-15 years. Food allergies that were eligible to be desensitized in this study were: cow's milk, eggs, peanuts, nuts, grains, and sesame seeds. As many as five allergen, all given in equal proportions, could be included in the treatment regimen. The initial rush OIT occurred at week 9, after 8 weeks of pre-treatment with omalizumab, with gradual escalation to a final dose of $1250 \mathrm{~g}$ protein (divided equally between different allergens). Doses were then increased every 2 weeks according to patient's tolerance of the previous home doses, without any fixed calendar. Omalizumab was stopped at week 16. The median time to reach the maintenance dose of $4000 \mathrm{mg}$ per allergen (up to $20,000 \mathrm{mg}$ if 5 foods were desensitized) was 18 weeks, with a range 7-36 weeks. By 2 months of therapy, all patients were able to tolerate a dose of allergen equivalent 
to a tenfold increase compared to their baseline levels, and all subjects were able to achieve maintenance dose by 9 months of therapy. This protocol proved itself to be relatively safe, as $96 \%$ of reactions that occurred were mild, and only one reaction was severe and warranted epinephrine administration. Moreover, reactions tended to occur with decreasing frequency over time, with a reaction rate that went down by $70 \%$ at 6 months of immunotherapy.

\section{Ongoing Studies}

The PRROTECT study (Peanut Reactivity Reduced by Oral Tolerance in an anti-IgE Clinical Trial, NCT01781637) is a double-blind, placebo-controlled clinical trial taking place in four centers: the Boston Children's Hospital, Stanford University, the Children's Hospital of Philadelphia, and Lurie Children's Hospital in Chicago. Participants are pre-treated with either omalizumab or placebo before oral peanut desensitization and continued for 2 months, and investigators will evaluate the ability of the subjects to successfully tolerate a rapid buildup phase (14 weeks) leading to a daily dose of $4000 \mathrm{mg}$ of peanut flour even after stopping anti-IgE therapy.

A second study evaluating the use of omalizumab with peanut OIT is also in progress (NCT00932282). Subjects receive a four-month pre-OIT therapy with omalizumab, and continue this therapy until 1 month after maintenance therapy, which should lead to $8000 \mathrm{mg}$ of peanut powder as maintenance dose. This trial will assess the safety, tolerability, and efficacy of omalizumab use in this context.

Finally, a third study is currently underway and seeks to evaluate the potential of omalizumab therapy in conjunction with milk OIT and subsequent food tolerance after having stopped OIT for 2 months (NCT01157117).

\section{Main Issues Regarding Omalizumab in OIT}

OIT trials using omalizumab seem to demonstrate many advantages over the conventional OIT, although many questions still remain unanswered.

\section{Benefits of Omalizumab}

\section{Higher Initial Dose than Conventional OIT}

In the peanut OIT trial using omalizumab, all 13 subjects $(100 \%)$ tolerated a cumulative peanut flour dose of $992 \mathrm{mg}$ on the initial OIT day, whereas in two other similar studies of oral desensitization, only 6/28 (21\%) (Hofmann et al. 2009) and 10/39 (26\%) (Jones et al. 2009), respectively, tolerated a cumulative dose equivalent to $200 \mathrm{mg}$ of peanut flour. This represents a four-to-fivefold increase in the percentage of patients being able to tolerate a cumulative dose almost five times higher, leading to faster protection against accidental exposures to small quantities of allergen. These findings are even more convincing considering that the median peanut-specific IgE levels in the omalizumab-assisted trial were much higher than in the aforementioned studies.

\section{Target Maintenance Dose Achieved Faster}

Adding omalizumab to OIT protocols led to faster achievement of the final maintenance doses when compared to the conventional OIT. For example, in a peanut oral desensitization study, it took a median time of 30 weeks to reach doses of 500-2000 mg of peanuts in 14 subjects (Blumchen et al. 2010) and a median time of 20 weeks for 19 subjects to reach the equivalent of $1600 \mathrm{mg}$ peanut flour (Anagnostou et al. 2011). In contrast, in the study by Schneider et al. (2013) combining omalizumab treatment to their OIT protocol, it took only a median of 8 weeks for 12 patients to tolerate $4000 \mathrm{mg}$ peanut flour.

In the study by Wood et al. (2016), which compared omalizumab-treated patients to placebo in milk OIT, the maintenance dose was reached significantly faster due to fewer OIT doses necessary to achieve the final maintenance dose (median 198.0 vs. $225 ; p=0.008$ ), leading to a shortened escalation phase (median 25.9 vs. 30.0 weeks; $p=0.01$ ).

Finally, in study by Begin et al. (2014a), using omalizumab in multiple foods desensitization, the median time for their subjects to reach the same maintenance dose of $4000 \mathrm{mg}$ per allergen was 67 weeks shorter than previously described in a similar study by the same author without the use of omalizumab.

\section{Safety of OIT: Less Reactions}

In the study by Wood et al. (2016), the benefits of using omalizumab in OIT were apparent on virtually all safety aspects. Patients were significantly less likely to have a reaction while being desensitized if they were on omalizumab compared to the placebo group, and tended to require less use of epinephrine (Wood et al. 2016). Unsurprisingly, patients who were at higher risk of moderate to severe reactions were those with a history of asthma and a higher milk-specific IgE baseline.

In study by Schneider et al. (2013), none of the severe reactions occurred, while patients were on omalizumab, which corroborates the idea that omalizumab might have a protective effect on such reactions. 
In another study using omalizumab with milk OIT, reactions occurred somewhat rarely (mean frequency of $1.6 \%$ of doses), and the majority of these reactions were mild (Nadeau et al. 2011). Moreover, none of the reactions occurred involved the cardiovascular system, and all reactions were responsive to treatment. However, there was no decrease in epinephrine use when compared to other studies. Safety still seems to be improved, as the previous studies without omalizumab have reported reaction rates as high as $45 \%$ of milk OIT doses, despite using lower initial and final maintenance doses (Skripak et al. 2008).

In Begin et al. (2014a) multiple allergens OIT study, reaction rates were comparable to those found in a similar study of patients undergoing OIT without omalizumab, although the former study used an accelerated desensitization schedule. Again, most of these reactions (96\%) were mild. The majority of these reactions occurred during the first month of OIT, while on omalizumab, which can be explained by the nature of the rush protocol. In this same study, the only use of epinephrine occurred while off omalizumab therapy.

While omalizumab does seem to provide a protective effect, there is a potential risk of breakthrough reactions once omalizumab is stopped, as was highlighted in Lafuente et al.'s (2014) case-series study, where the recurrence of allergic reactions occurred after discontinuation of omalizumab. However, these patients had previously failed standard OIT and may not be representative of all OIT candidates. In Begin et al.'s study, there was a temporary increase in dosing reactions 2 months after omalizumab discontinuation, which subsequently trended back downwards (Begin et al. 2014a). The frequency, severity, and risk factors for breakthrough reactions need to be better documented in future studies.

\section{Safety of Omalizumab}

Omalizumab has proven itself to be relatively safe, with most reactions occurring locally at the injection site (Berger et al. 2003; Corren et al. 2009). More serious reactions, such as anaphylaxis, including delayed-onset anaphylaxis, have been reported. Close monitoring of patients during and following infusion is, therefore, warranted (Corren et al. 2009; Cox et al. 2007, 2011). Side effects reported in omalizumab-enabled OIT trials included injection site pain and swelling (Schneider et al. 2013), with no allergic reactions reported (Nadeau et al. 2011).

\section{Long-Term Tolerance}

Sustained unresponsiveness is defined by a state, where subjects can tolerate a food that they were previously allergic to after cessation of immunotherapy for about
4 weeks. The term immunological tolerance is used once the nonresponsiveness becomes permanent (Chhiba et al. 2015). The present studies have not yet shown an advantage attributable to omalizumab in creating a sustained unresponsiveness state after oral food desensitization. While Wood et al.'s study was unable to show this benefit, there was a trend towards this effect. Assuming that this difference of $12.4 \%$ was real, their study would have been underpowered to detect it (Wood et al. 2016). It would have required a sample size of 248 to do so. More studies are thus needed to answer this question with one currently underway (Hugh Samson, NCT01157117).

It is theoretically plausible that omalizumab could help create a more permanent tolerance to allergens considering the important pharmacological effects other than IgE sequestration that were discovered (Fig. 1). Animal models have showed that anti-IgE treatment causes downregulation of the high-affinity FceRI receptors on basophils, mast cells but also dendritic cells (DC) (Chang and Shiung 2006). Omalizumab could, therefore, suppress cellular response by inhibiting antigen presentation by dendritic cells to T cells (Holgate et al. 2005). This could also occur through basophils, as they also have a role in Th2 inflammatory disorders through direct interactions with pathogenic CD4 T cells as well as by enhancing DC-induced Th2 cell development (Wakahara et al. 2013). A recent study showed that when an adjuvant-free model of peanut allergy consisting of mice harboring a disinhibited form of the IL-4 receptor was crossed with IgE-deficient strains, not only did it suppress mast cell degranulation and anaphylaxis, but the generation of a Th2 response to food allergens in the gut was also dramatically reduced (Burton et al. 2014). Simultaneously, regulatory T cells (Tregs) induction, which was impaired in the allergy-prone mice, was fully restored in the absence of IgE. When combined with peanut oral immunotherapy (adapted protocol for mice), the administration of anti-IgE blockade mimicked genetic depletion of IgE. The reduction in Th2 cells and expansion of Tregs observed in combined anti-IgE and oral immunotherapy were not seen with oral immunotherapy alone.

Another important potential effect of anti-IgE treatment is the direct inhibition of $\mathrm{IgE}$ production by B cells. In vitro and in vivo studies have showed that anti-IgE antibodies bind to membrane bound $\operatorname{IgE}(\mathrm{mIgE})$, which is part of the $\mathrm{B}$ cell receptor, thus inhibiting the production of $\operatorname{IgE}$ and even cause cell lysis of IgE secreting B cells (Chang 2000). Although already established bone-marrow residing plasmocytes secreting IgE do not express mIgE and are not targeted by omalizumab, memory B cells and new IgE secreting plasma cells express mIgE and could therefore be affected. This could theoretically shift the long-term immune response from an $\mathrm{IgE}$ secreting $\mathrm{Th} 2$ profile to a 


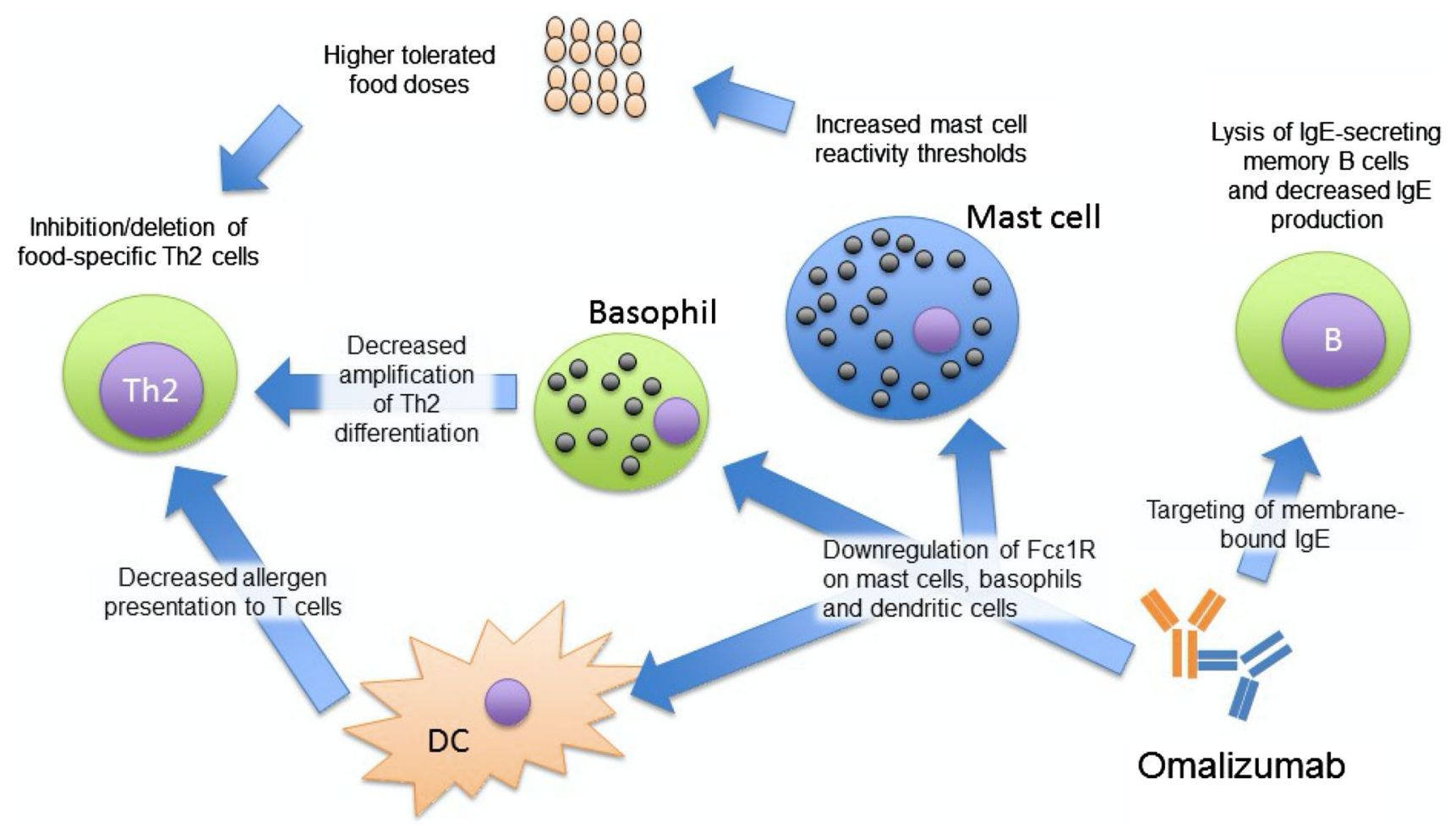

Fig. 1 Potentially relevant immunodulatory mechanisms of omalizumab to induce sustained tolerance in food oral immunotherapy

non-allergic phenotype (Chang 2000; Chang and Shiung 2006).

\section{Pharmacoeconomic Considerations}

Pharmacoeconomic analysis of omalizumab in OIT is complex, because the cost and benefit of OIT itself have not been fully established. The cost of OIT visits will vary depending on country, clinic structure, insurance plans, and public coverage. The cost of omalizumab depends on the dosage and duration of treatment. In addition to the cost of OIT visits saved by omalizumab, one should also take into account absenteeism from work from patients or their primary caregivers, which also represents significant expenses. Omalizumab may not be necessary for all patients undergoing OIT, and studies looking at a targeted use for allergic patients with lower allergy threshold or previous OIT failure are needed to optimize its use.

\section{Improved QoL}

The conventional single-allergen OIT has already been shown to significantly improve the quality of life of its participants (Carraro et al. 2012; Factor et al. 2012). Recently, Otani et al. (2014) evaluated the effects on QoL of the conventional multiple-allergen OIT and rush multiple-allergen OIT with anti-IgE treatment (omalizumab) when compared to allergic non-treated patients. All the three groups had a poor QoL at baseline, and both the treated groups had a substantial improvement in their QoL when evaluated throughout their therapy. At 6 months follow-up, the proportion of subjects with an increased QoL was higher in the group having received rush OIT with omalizumab when compared to the other OIT group (91 vs. $66 \%$ ), presumably due to faster achievement of maintenance dose. Moreover, the extent of this improvement was significantly greater in the omalizumab-treated group, both clinically and statistically.

Another report of a patient receiving OIT in combination with omalizumab demonstrated an improvement of his quality of life (Takahashi et al. 2015).

These results suggest that, from a caregiver standpoint, the reactions that can potentially occur with OIT are better perceived than allergen avoidance, and the quality of life can be improved faster in rush OIT with omalizumab pretreatment.

\section{Optimal Use of Omalizumab}

\section{Timing of Omalizumab Use Pre-OIT}

The half-life of circulating IgE is $24-48 \mathrm{~h}$. However, IgE molecules stay attached to the FceRI on mastocytes during weeks to months (Achatz et al. 2008), which is why omalizumab needs to be given several weeks before starting OIT to be effective. 
Although the previous studies suggest that 16 weeks of omalizumab treatment might be necessary before obtaining a clinical response in asthmatic patients (Bousquet et al. 2004), 8 weeks might be sufficient for optimal response when used in OIT. A study led by Jessica Savage showed a 56-fold increase in the peanut challenge dose in peanutallergic patients after only 8 weeks of omalizumab, with a similar response after 6 months of treatment (Savage et al. 2012). Ex vivo studies on basophil expression of CD203c, which is thought to be a marker of allergen-dependent basophil activation, also showed a marked decrease in basophil CD203c levels after 8 weeks of omalizumab therapy in subjects with nut allergy (Gernez et al. 2011). To date, most trials have used a pre-OIT treatment with omalizumab ranging from 8-12 weeks, with only one using over four months of therapy prior to OIT (Wood et al. 2016), the rationale behind the use of a longer course not being specified by the authors.

\section{Length of Omalizumab Therapy}

The overall length of omalizumab therapy in OIT trials has varied from a minimum of 16 weeks to over 2 years. To this day, the ideal length of omalizumab therapy has yet to be determined. As previously discussed, patients seemed to be protected from severe reactions while on omalizumab therapy (Schneider et al. 2013), and some patients experience recurrence of symptoms once omalizumab is stopped (Begin et al. 2014a; Lafuente et al. 2014). Optimal length of omalizumab use needs to evaluate the cost-benefit value behind a shorter use in rush protocol with a maintenance phase achieved faster compared to longer use that could potentially prevent serious reactions from occurring.

\section{Ideal Moment for Challenge}

Given that the half-life of omalizumab is approximately 2 weeks (Chang 2000), a washout of the molecule occurs 2-3 months after discontinuing therapy. Although most patients in OIT trials were challenged 2-3 months after omalizumab therapy was ceased with few significant reactions (Nadeau et al. 2011, Schneider et al. 2013, Wood et al. 2016), Lafuente et al.'s (2014) report suggests that patients might be susceptible to developing reactions later, around 3-4 months after stopping omalizumab.

\section{Combination with Other Immunomodulatory Approaches}

Although unexpected pharmacological benefits have been discovered with omalizumab use, the main effect of antiIgE therapy remains modulation of humoral immunity. Newer immunomodulators targeting cytokines are emerging, of which dupilumab is of particular interest in allergic diseases. It has already shown promising results in atopic dermatitis, asthma, and chronic rhinosinusitis (Beck et al. 2014; Hamilton et al. 2014; McGregor et al. 2015; Pauwels et al. 2015; Wenzel et al. 2013). Dupilumab is an anti-IL$4 \alpha$ receptor, which is shared by both Th2-derived cytokines IL-4 and IL-13, thus modulating the cellular immunologic response in allergic diseases (Andrews et al. 2006). It could, therefore, potentially play a role in sustained tolerance. The combination of both therapies could allow for complementary effects in OIT: the use of omalizumab to safely accelerate build-up phase, followed by dupilumab therapy during maintenance phase to hopefully promote long-term tolerance. Nevertheless, the safety and efficacy of combining these molecules have yet to be studied. Appropriate pre-clinical and clinical trials need to be done before such a combination can be used more broadly.

\section{Conclusion}

Omalizumab is a promising adjunctive therapy for oral immunotherapy. Studies performed with omalizumab indicate a faster escalation period with less adverse reactions and improved the quality of life. All the data currently available on omalizumab are based on small studies, most of them cohort studies, and therefore, future and currently ongoing studies will provide important information regarding the actual benefits of omalizumab in food OIT. These studies will help to expand clinical experience by determining optimal doses and timing of omalizumab therapy, and eventually clear the way for combination immunomodulatory approaches.

\section{Compliance with ethical standards}

Conflict of interest The authors declare that they have no conflict of interest.

\section{References}

Achatz G, Lamers M, Crameri R (2008) Membrane bound IgE-the key receptor to restrict high IgE levels. Open Immunol J 1:25-32

Anagnostou K, Clark A, King Y et al (2011) Efficacy and safety of high-dose peanut oral immunotherapy with factors predicting outcome. Clin Exp Allergy 41:1273-1281

Andrews AL, Holloway JW, Holgate ST et al (2006) IL-4 receptor alpha is an important modulator of IL-4 and IL-13 receptor binding: implications for the development of therapeutic targets. J Immunol 176:7456-7461

Beck LA, Thaci D, Hamilton JD et al (2014) Dupilumab treatment in adults with moderate-to-severe atopic dermatitis. N Engl J Med 371:130-139

Begin P, Dominguez T, Wilson SP et al (2014a) Phase 1 results of safety and tolerability in a rush oral immunotherapy protocol to multiple foods using omalizumab. Allergy Asthma Clin Immunol 10:7 
Begin P, Winterroth LC, Dominguez T et al (2014b) Safety and feasibility of oral immunotherapy to multiple allergens for food allergy. Allergy Asthma Clin Immunol 10:1

Ben-Shoshan M, Kagan RS, Alizadehfar R et al (2009) Is the prevalence of peanut allergy increasing? A 5-year follow-up study in children in Montreal. J Allergy Clin Immunol 123:783-788

Berger W, Gupta N, McAlary M et al (2003) Evaluation of long-term safety of the anti-IgE antibody, omalizumab, in children with allergic asthma. Ann Allergy Asthma Immunol 91:182-188

Beyer K, Wahn U (2008) Oral immunotherapy for food allergy in children. Curr Opin Allergy Clin Immunol 8:553-556

Blumchen K, Ulbricht H, Staden U et al (2010) Oral peanut immunotherapy in children with peanut anaphylaxis. J Allergy Clin Immunol 126:83-91.e1

Bock SA, Munoz-Furlong A, Sampson HA (2001) Fatalities due to anaphylactic reactions to foods. J Allergy Clin Immunol 107:191-193

Bousquet J, Wenzel S, Holgate S et al (2004) Predicting response to omalizumab, an anti-IgE antibody, in patients with allergic asthma. Chest 125:1378-1386

Boyce JA, Assa'ad A, Burks AW et al (2010) Guidelines for the diagnosis and management of food allergy in the United States: summary of the NIAID-sponsored expert panel report. J Allergy Clin Immunol 126:105-1118

Buchanan AD, Green TD, Jones SM et al (2007) Egg oral immunotherapy in nonanaphylactic children with egg allergy. J Allergy Clin Immunol 119:199-205

Burks AW, Jones SM, Wood RA et al (2012) Oral immunotherapy for treatment of egg allergy in children. N Engl J Med 367:233-243

Burton OT, Noval Rivas M, Zhou JS et al (2014) Immunoglobulin E signal inhibition during allergen ingestion leads to reversal of established food allergy and induction of regulatory $\mathrm{T}$ cells. Immunity 41:141-151

Carraro S, Frigo AC, Perin $M$ et al (2012) Impact of oral immunotherapy on quality of life in children with cow milk allergy: a pilot study. Int $\mathrm{J}$ Immunopathol Pharmacol 25:793-798

Chang TW (2000) The pharmacological basis of anti-IgE therapy. Nat Biotechnol 18:157-162

Chang TW, Shiung YY (2006) Anti-IgE as a mast cell-stabilizing therapeutic agent. J Allergy Clin Immunol 117:1203-1212 (quiz 1213)

Chhiba KD, Singh AM, Bryce PJ (2015) New developments in immunotherapies for food allergy. Immunotherapy 7:913-922

Clark AT, Islam S, King Y et al (2009) Successful oral tolerance induction in severe peanut allergy. Allergy 64:1218-1220

Corren J, Casale TB, Lanier B et al (2009) Safety and tolerability of omalizumab. Clin Exp Allergy 39:788-797

Cox L, Platts-Mills TA, Finegold I et al (2007) American Academy of Allergy, Asthma and Immunology/American College of Allergy, Asthma and Immunology Joint Task Force Report on omalizumab-associated anaphylaxis. J Allergy Clin Immunol 120:1373-1377

Cox L, Lieberman P, Wallace D et al (2011) American Academy of Allergy, Asthma and Immunology/American College of Allergy, Asthma and Immunology Omalizumab-Associated Anaphylaxis Joint Task Force follow-up report. J Allergy Clin Immunol 128:210-212

Cummings AJ, Knibb RC, King RM et al (2010) The psychosocial impact of food allergy and food hypersensitivity in children, adolescents and their families: a review. Allergy 65:933-945

Eggesbo M, Botten G, Halvorsen R et al (2001) The prevalence of allergy to egg: a population-based study in young children. Allergy 56:403-411
Factor JM, Mendelson L, Lee J et al (2012) Effect of oral immunotherapy to peanut on food-specific quality of life. Ann Allergy Asthma Immunol 109(348-352):e2

Garcia-Ara MC, Boyano-Martinez MT, Diaz-Pena JM et al (2004) Cow's milk-specific immunoglobulin E levels as predictors of clinical reactivity in the follow-up of the cow's milk allergy infants. Clin Exp Allergy 34:866-870

Gernez Y, Tirouvanziam R, Yu G et al (2011) Basophil CD203c levels are increased at baseline and can be used to monitor omalizumab treatment in subjects with nut allergy. Int Arch Allergy Immunol 154:318-327

Gupta RS, Springston EE, Warrier MR et al (2011) The prevalence, severity, and distribution of childhood food allergy in the United States. Pediatrics 128:e9-17

Gupta R, Holdford D, Bilaver L et al (2013) The economic impact of childhood food allergy in the United States. JAMA Pediatr 167:1026-1031

Hamilton JD, Suarez-Farinas M, Dhingra N et al (2014) Dupilumab improves the molecular signature in skin of patients with moderate-to-severe atopic dermatitis. J Allergy Clin Immunol 134:1293-1300

Hofmann AM, Scurlock AM, Jones SM et al (2009) Safety of a peanut oral immunotherapy protocol in children with peanut allergy. J Allergy Clin Immunol 124:286-291, e1-6

Holgate ST, Djukanovic R, Casale T et al (2005) Anti-immunoglobulin $\mathrm{E}$ treatment with omalizumab in allergic diseases: an update on anti-inflammatory activity and clinical efficacy. Clin Exp Allergy 35:408-416

Jagdis A, Berlin N, Barron C et al (2014) Effect of ketotifen premedication on adverse reactions during peanut oral immunotherapy. Allergy Asthma Clin Immunol 10:36

Jones SM, Pons L, Roberts JL et al (2009) Clinical efficacy and immune regulation with peanut oral immunotherapy. J Allergy Clin Immunol 124:292-300, 300.e1-97

Lafuente I, Mazon A, Nieto M et al (2014) Possible recurrence of symptoms after discontinuation of omalizumab in anti-IgE-assisted desensitization to egg. Pediatr Allergy Immunol 25:717-719

Lanser BJ, Wright BL, Orgel KA et al (2015) Current options for the treatment of food allergy. Pediatr Clin N Am 62:1531-1549

Leung DY, Sampson HA, Yunginger JW et al (2003) Effect of antiIgE therapy in patients with peanut allergy. N Engl J Med 348:86-993

Longo G, Barbi E, Berti I et al (2008) Specific oral tolerance induction in children with very severe cow's milk-induced reactions. J Allergy Clin Immunol 121:343-347

McGowan EC, Keet CA (2013) Prevalence of self-reported food allergy in the National Health and Nutrition Examination Survey (NHANES) 2007-2010. J Allergy Clin Immunol 132:12161219.e5

McGregor S, Farhangian ME, Feldman SR (2015) Dupilumab for the treatment of atopic dermatitis: a clinical trial review. Expert Opin Biol Ther 15:1657-1660

Meglio P, Bartone E, Plantamura M et al (2004) A protocol for oral desensitization in children with IgE-mediated cow's milk allergy. Allergy 59:980-987

Nadeau KC, Schneider LC, Hoyte L et al (2011) Rapid oral desensitization in combination with omalizumab therapy in patients with cow's milk allergy.". J Allergy Clin Immunol 127:1622-1624

Otani IM, Begin P, Kearney C et al (2014) Multiple-allergen oral immunotherapy improves quality of life in caregivers of foodallergic pediatric subjects. Allergy Asthma Clin Immunol 10:25

Patriarca G, Nucera E, Roncallo C et al (2003) Oral desensitizing treatment in food allergy: clinical and immunological results. Aliment Pharmacol Ther 17:459-465 
Pauwels B, Jonstam K, Bachert C (2015) Emerging biologics for the treatment of chronic rhinosinusitis. Expert Rev Clin Immunol 11:349-361

Protudjer JL, Jansson SA, Ostblom E et al (2015) Health-related quality of life in children with objectively diagnosed staple food allergy assessed with a disease-specific questionnaire. Acta Paediatr 104:1047-1054

Sampson HA, Leung DY, Burks AW et al (2011) A phase II, randomized, doubleblind, parallelgroup, placebocontrolled oral food challenge trial of Xolair (omalizumab) in peanut allergy. J Allergy Clin Immunol 127(1309-1310):e1

Sato S, Yanagida N, Ogura K et al (2014) Clinical studies in oral allergen-specific immunotherapy: differences among allergens. Int Arch Allergy Immunol 164:1-9

Savage JH, Courneya JP, Sterba PM et al (2012) Kinetics of mast cell, basophil, and oral food challenge responses in omalizumabtreated adults with peanut allergy. J Allergy Clin Immunol 130(1123-1129):e2

Schneider LC, Rachid R, LeBovidge J et al (2013) A pilot study of omalizumab to facilitate rapid oral desensitization in high-risk peanut-allergic patients. J Allergy Clin Immunol 132:1368-1374

Segal M, Stokes JR, Casale TB (2008) Anti-immunoglobulin e therapy. World Allergy Organ J 1:174-183

Shek LP, Soderstrom L, Ahlstedt S et al (2004) Determination of food specific IgE levels over time can predict the development of tolerance in cow's milk and hen's egg allergy. J Allergy Clin Immunol 114:387-391

Sicherer SH (2011) Epidemiology of food allergy. J Allergy Clin Immunol 127:594-602

Sicherer SH, Sampson HA (2010) Food allergy. J Allergy Clin Immunol 125(2 Suppl 2):S116-S125

Sicherer SH, Noone SA, Munoz-Furlong A (2001) The impact of childhood food allergy on quality of life. Ann Allergy Asthma Immunol 87:461-464

Sicherer SH, Munoz-Furlong A, Sampson HA (2003) Prevalence of peanut and tree nut allergy in the United States determined by means of a random digit dial telephone survey: a 5-year followup study. J Allergy Clin Immunol 112:1203-1207

Skolnick HS, Conover-Walker MK, Koerner CB et al (2001) The natural history of peanut allergy. J Allergy Clin Immunol 107:367-374

Skripak JM, Nash SD, Rowley H et al (2008) A randomized, doubleblind, placebo-controlled study of milk oral immunotherapy for cow's milk allergy. J Allergy Clin Immunol 122:1154-1160
Soller L, Ben-Shoshan M, Harrington DW et al (2012) Overall prevalence of self-reported food allergy in Canada. J Allergy Clin Immunol 130:986-988

Sova C, Feuling MB, Baumler M et al (2013) Systematic review of nutrient intake and growth in children with multiple IgEmediated food allergies. Nutr Clin Pract 28:669-675

Staden U, Rolinck-Werninghaus C, Brewe F et al (2007) Specific oral tolerance induction in food allergy in children: efficacy and clinical patterns of reaction. Allergy 62:1261-1269

Staden U, Blumchen K, Blankenstein N et al (2008) Rush oral immunotherapy in children with persistent cow's milk allergy. J Allergy Clin Immunol 122:418-419

Takahashi M, Taniuchi S, Soejima K et al (2014) New efficacy of LTRAs (montelukast sodium): it possibly prevents food-induced abdominal symptoms during oral immunotherapy. Allergy Asthma Clin Immunol 10:3

Takahashi M, Taniuchi S, Soejima K et al (2015) Successful desensitization in a boy with severe cow's milk allergy by a combination therapy using omalizumab and rush oral immunotherapy. Allergy Asthma Clin Immunol 11:18

Umetsu DT, Rachid R, Schneider LC (2015) Oral immunotherapy and anti-IgE antibody treatment for food allergy. World Allergy Organ J 8:20

Varshney P, Jones SM, Scurlock AM et al (2011) A randomized controlled study of peanut oral immunotherapy: clinical desensitization and modulation of the allergic response. J Allergy Clin Immunol 127:654-660

Wakahara K, Van VQ, Baba N et al (2013) Basophils are recruited to inflamed lungs and exacerbate memory $\mathrm{Th} 2$ responses in mice and humans. Allergy 68:180-189

Wenzel S, Ford L, Pearlman D et al (2013) Dupilumab in persistent asthma with elevated eosinophil levels. $\mathrm{N}$ Engl J Med 368:2455-2466

Wood RA, Kim JS, Lindblad R et al (2016) A randomized, doubleblind, placebo-controlled study of omalizumab combined with oral immunotherapy for the treatment of cow's milk allergy. J Allergy Clin Immunol 137(1103-1110):e11

Wright JD, Chu HM, Huang CH et al (2015) Structural and physical basis for anti-IgE therapy. Sci Rep 5:11581

Yu JW, Kagan R, Verreault N et al (2006) Accidental ingestions in children with peanut allergy. J Allergy Clin Immunol 118:466-472 